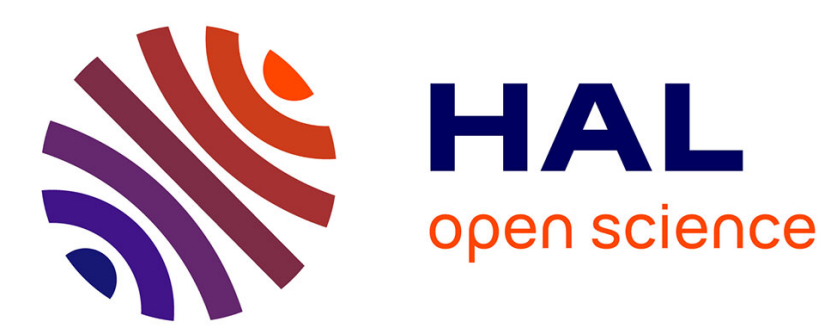

\title{
Role of Fourier Modes in Finite-Size Scaling above the Upper Critical Dimension.
}

Emilio José Flores-Sola, Bertrand Berche, Ralph Kenna, Martin Weigel

\section{To cite this version:}

Emilio José Flores-Sola, Bertrand Berche, Ralph Kenna, Martin Weigel. Role of Fourier Modes in Finite-Size Scaling above the Upper Critical Dimension.. Physical Review Letters, 2016, 116, 10.1103/PhysRevLett.116.115701 . hal-01298202

\section{HAL Id: hal-01298202 \\ https://hal.science/hal-01298202}

Submitted on 3 Mar 2017

HAL is a multi-disciplinary open access archive for the deposit and dissemination of scientific research documents, whether they are published or not. The documents may come from teaching and research institutions in France or abroad, or from public or private research centers.
L'archive ouverte pluridisciplinaire HAL, est destinée au dépôt et à la diffusion de documents scientifiques de niveau recherche, publiés ou non, émanant des établissements d'enseignement et de recherche français ou étrangers, des laboratoires publics ou privés. 


\title{
Role of Fourier Modes in Finite-Size Scaling above the Upper Critical Dimension
}

\author{
Emilio Flores-Sola, ${ }^{1,2,3}$ Bertrand Berche, ${ }^{2,3}$ Ralph Kenna, ${ }^{1,3}$ and Martin Weigel ${ }^{1,3}$ \\ ${ }^{1}$ Applied Mathematics Research Centre, Coventry University, Coventry CV1 5FB, United Kingdom \\ ${ }^{2}$ Institut Jean Lamour, CNRS/UMR 7198, Groupe de Physique Statistique, Université de Lorraine, \\ BP 70239, F-54506 Vandouvre-les-Nancy Cedex, France \\ ${ }^{3}$ Doctoral College for the Statistical Physics of Complex Systems, Leipzig-Lorraine-Lviv-Coventry $\left(\mathbb{L}^{4}\right)$, D-04009 Leipzig, Germany
}

(Received 28 October 2015; published 18 March 2016)

\begin{abstract}
Renormalization-group theory has stood, for over 40 years, as one of the pillars of modern physics. As such, there should be no remaining doubt regarding its validity. However, finite-size scaling, which derives from it, has long been poorly understood above the upper critical dimension $d_{c}$ in models with free boundary conditions. In addition to its fundamental significance for scaling theories, the issue is important at a practical level because finite-size, statistical-physics systems with free boundaries and above $d_{c}$ are experimentally relevant for long-range interactions. Here, we address the roles played by Fourier modes for such systems and show that the current phenomenological picture is not supported for all thermodynamic observables with either free or periodic boundaries. In particular, the expectation that dangerous irrelevant variables cause Gaussian-fixed-point scaling indices to be replaced by Landau mean-field exponents for all Fourier modes is incorrect. Instead, the Gaussian-fixed-point exponents have a direct physical manifestation for some modes above the upper critical dimension.
\end{abstract}

DOI: 10.1103/PhysRevLett.116.115701

In this Letter we address a subtle question, still open, in the theory of finite-size scaling (FSS). Although it may cursorily appear to be an academic exercise, this question has to be decisively and convincingly resolved since it concerns the very foundations of the renormalization group (RG), one of the greatest achievements of theoretical physics [1]. Given the nature of our investigation, and with the aim of a comprehensive presentation, we emphasize essential historical and relatively technical elements of the theory before introducing the problem of Fourier modes in the treatment of boundary conditions.

FSS is a well developed component of modern theories of critical phenomena. It was initially introduced on heuristic grounds [2] and then understood within the frame of Wilson's RG [1,3-5]. The system's inverse typical linear scale $L^{-1}$ appears like the reduced temperature $t=\left(T-T_{c}\right) / T_{c}$ or the magnetic field $h=H / T_{c}$ as a scaling field governing flow towards the RG fixed points (FPs) which control the critical singularities of thermodynamic properties,

$$
\begin{aligned}
C(t) & \sim|t|^{-\alpha}, & m(t) \underset{t<0}{\sim}|t|^{\beta}, &
\end{aligned}
$$

Here, $C, m, \chi, \xi$, and $g$ are, respectively, the singular parts of the specific heat, magnetization, susceptibility, correlation length, and correlation function, and the fields $t$ or $h$, when not specified, are zero. The long-distance properties at the FPs depend on space dimension $d$ and order-parameter symmetry, but not on details such as the short range of microscopic interactions, or lattice symmetry. Hence, there exists a set of properties (the critical exponents as well as combinations of critical amplitudes not considered here) which rigorously take the same values for different systems. Magnetic systems, for example, can share these properties with fluids. This is the meaning of the term "universality" used in this context [6].

The usual phenomenological argument for FSS (which turns out to be valid only below the upper critical dimension) can be summarized as follows [2,5]. Consider a quantity $P\left(t, L^{-1}\right)$ that, for $L \rightarrow \infty$, exhibits a singularity in the vicinity of the critical point $T_{c}$, measured by a critical exponent $\rho$, so that $P(t, 0) \sim|t|^{\rho}$. Then, this singularity develops in a finite-size system as

$$
P\left(t, L^{-1}\right) \sim P(t, 0) \mathcal{P}[L / \xi(t)],
$$

where, it is argued, the ratio $L / \xi(t)$ appears because it involves the only two length scales governing long-distance behavior, and the scaling function $\mathcal{P}$ exhibits a power-law behavior $\mathcal{P}(z) \sim z^{-\rho / \nu}$, such that the singularity in $t$ is washed out at $T_{c}$ for the finite system. This argument predicts FSS exponents $\gamma / \nu$ for the susceptibility, and $-\beta / \nu$ for the magnetization.

A spectacular result of Wilson's RG is the explanation for the existence of an upper critical dimension $d_{c}$ above which Landau mean-field theory (MFT) is recovered, with

$$
\begin{array}{lll}
\alpha=0, & \beta=1 / 2, & \delta=3, \\
\gamma=1, & \nu=1 / 2, & \eta=0
\end{array}
$$

for $\phi^{4}$-field theory. To fix ideas, let us consider a nearestneighbor Ising model consisting of spins $s_{\mathbf{x}}$ located on the sites $\mathbf{x}$ of a regular hypercubic lattice with unit vectors $\boldsymbol{\mu}$ $(|\boldsymbol{\mu}|=a$, the lattice spacing). The exact partition function 


$$
Z=\sum_{\left\{s_{\mathbf{x}}\right\}} e^{\beta\left(J \sum_{\mathbf{x}} \sum_{\mu} s_{\mathbf{x}} s_{\mathbf{x}+\mu}+H \sum_{\mathbf{x}} s_{\mathbf{x}}\right)}
$$

can be rewritten via a Hubbard-Stratonovich transformation as a functional integral over $\phi(\mathbf{x}) \in \mathbb{R}$, $Z \simeq \prod_{\mathbf{x}} \int d \phi(\mathbf{x}) e^{-S[\phi]}$, with

$S[\phi]=\int d^{d} x\left(f_{0}+\frac{r_{0}}{2} \phi^{2}+\frac{u}{4} \phi^{4}+\frac{c}{2}|\nabla \phi|^{2}-h \phi\right)$,

where $f_{0}=-a^{-d} \ln 2, r_{0}=a^{-2} t, u=2 a^{d-4}, c=(2 d)^{-1}$, and $h=\beta a^{-(d / 2+1)} H$. Dimensional analysis shows that the free energy $f_{0}$ has scaling dimension $d$, the coefficient $r_{0}$, proportional to the reduced temperature, has dimension $y_{t}=2$, the coefficient of the quartic term $u$ has $y_{u}=4-d$, and the magnetic field has $y_{h}=d / 2+1$. The eigenvalues $y_{i}>0$ control the flow of the relevant fields at the FP, leading to homogeneity:

$$
\begin{gathered}
f(t, h)=b^{-d} F\left(b^{y_{t}} t, b^{y_{h}} h\right), \\
\xi(t, h)=b \Xi\left(b^{y_{t}} t, b^{y_{h}} h\right), \\
g(t, h, \mathbf{x})=b^{-d+2} G\left(b^{y_{t}} t, b^{y_{h}} h, b^{-1} \mathbf{x}\right) .
\end{gathered}
$$

The critical exponents in Eqs. (1) and (2) emerge through the scaling laws,

$\alpha=\left(2 y_{t}-d\right) / y_{t}, \quad \beta=\left(d-y_{h}\right) / y_{t}, \quad \delta=y_{h} /\left(d-y_{h}\right)$,

$\gamma=\left(2 y_{h}-d\right) / y_{t}, \quad \nu=1 / y_{t}, \quad \eta=d-2 y_{h}+2$.

Above $d_{c}=4, y_{u}<0$, and one expects that critical behavior should be controlled by the Gaussian FP $(t, h, u)=(0,0,0)$ and characterized by these exponents. However, a discrepancy between the MFT exponents in Eqs. (4) and (11) indicates that the limit $u \rightarrow 0$ has to be taken with care; $u$ is a dangerous irrelevant variable (DIV) [7], at least, as was first thought, in the free-energy sector, the agreement between Eqs. (5) and (12) suggesting that there is no danger in the sector of the correlations [8]. A proper inclusion of this mechanism resolves the discrepancy in the free-energy sector. In Fourier space, for a periodic system, the quadratic part of the action (7) reads $\frac{1}{2} \sum_{\mathbf{k}}\left(|\mathbf{k}|^{2}+r_{0}\right)\left|\phi_{\mathbf{k}}\right|^{2}$, and the quartic term $\frac{1}{4} u \int d^{d} x \phi^{4}(\mathbf{x})=$ $\left(u / 4 L^{d}\right) \sum_{\mathbf{k}_{1} \mathbf{k}_{2} \mathbf{k}_{3}} \phi_{\mathbf{k}_{1}} \phi_{\mathbf{k}_{2}} \phi_{\mathbf{k}_{3}} \phi_{-\mathbf{k}_{1}-\mathbf{k}_{2}-\mathbf{k}_{3}}$ can be expanded as $\frac{1}{4}\left(u / L^{d}\right) \phi_{0}^{4}+\frac{3}{2}\left(u / L^{d}\right) \phi_{0}^{2} \sum_{\mathbf{k}}\left|\phi_{\mathbf{k}}\right|^{2}$ up to higher-order corrections in the nonzero modes, such that the action can be approximated by

$$
\begin{aligned}
S[\phi] \simeq & \frac{1}{2}\left(r_{0}+\frac{3 u}{2 L^{d}} \sum_{\mathbf{k} \neq 0}\left|\phi_{\mathbf{k}}\right|^{2}\right) \phi_{0}^{2}+\frac{u}{4 L^{d}} \phi_{0}^{4} \\
& +\frac{1}{2} \sum_{\mathbf{k} \neq 0}\left(r_{0}+c|\mathbf{k}|^{2}\right)\left|\phi_{\mathbf{k}}\right|^{2}-h L^{d / 2} \phi_{0} .
\end{aligned}
$$

Despite its irrelevance, $u$ is dangerous [7] for the zero modes above $d_{c}$. The nonzero modes do not have this feature.

The zero mode is thus responsible for anomalous FSS behavior above $d_{c}$ and leads to

$f\left(t, u, h, L^{-1}\right)=b^{-d} F\left(\frac{b^{y_{t}} t-b^{y_{u}} u}{b^{y_{u} / 2} u^{1 / 2}}, \frac{b^{y_{h}} h}{b^{y_{u} / 4} u^{1 / 4}}, \frac{b}{L}\right)$.

The temperature field $t$ is governed by a modified RG exponent $y_{t}^{*}=y_{t}-y_{u} / 2=d / 2$, and the magnetic field $h$ by $y_{h}^{*}=y_{h}-y_{u} / 4=3 d / 4$ [9-11]. Temperature dependencies of the magnetization and susceptibility follow by differentiating (14) with respect to $h$ and choosing the scale factor $b=\left(t / u^{1 / 2}\right)^{-2 / d}$. Hence,

$$
\begin{aligned}
& m(t, u, 0)=B(u) t^{1 / 2} M\left[1-A(u) t^{1-4 / d}\right], \\
& \chi(t, u, 0)=\Gamma(u) t^{-1} X\left[1-A(u) t^{1-4 / d}\right]
\end{aligned}
$$

and we now obtain the correct MFT exponents above $d_{c}$. [We omit here the specific heat and critical isotherm for which the same argument holds; Eqs. (11) and (12) deliver all mean-field exponents with $y_{t}^{*}$ and $y_{h}^{*}$ in place of the original scaling dimensions]. The finite-size behavior is immediate by setting $b=L$ in Eq. (14) and differentiating appropriately [12], e.g.,

$$
\begin{aligned}
& m_{T_{c}}\left(u, L^{-1}\right) \sim L^{-d / 4} M\left(A L^{2-d / 2} u^{1 / 2}\right), \\
& \chi_{T_{c}}\left(u, L^{-1}\right) \sim L^{d / 2} X\left(A L^{2-d / 2} u^{1 / 2}\right) .
\end{aligned}
$$

If the finite-size correlation length were bounded by the system length [12], one could not write the combination $L^{y_{t}^{*}} t$, which enters the free energy, as a ratio $L / \xi$ along the lines of Eq. (3). Within this framework, another length scale $\ell(t)$ was introduced, dubbed the thermodynamic length, with $\ell(t) \sim t^{-2 / d}$, with FSS being governed by the ratio $L / \ell(t)$ instead [13].

Contrary to previously widespread opinion [8,12-17], the correlation sector also needs reexamination and the homogeneity assumption above $d_{c}$ there takes the form [18]

$$
\begin{aligned}
\xi(t, u) & =b^{\varrho} \Xi\left(b^{d / 2} \frac{t}{u^{1 / 2}}-A b^{2-d / 2} u^{1 / 2}\right), \\
g(t, u, \mathbf{x}) & =b^{-d / 2} G\left(b^{d / 2} \frac{t}{u^{1 / 2}}-A b^{2-d / 2} u^{1 / 2}, b^{-1} \mathbf{x}\right),
\end{aligned}
$$

where we omit the $h$ dependence for clarity. Here, the exponent $Q$ ("koppa") extends the DIV mechanism to the 
correlation length [19]. This leads to a new interpretation, dubbed QFSS in Ref. [19], above $d=d_{c}$ dimensions. [The $Q$ refers to $Q$, which governs the FSS of the correlation length in Eq. (19)]. The finite-size behavior is transparent from Eqs. (19) and (20); fixing the scale factor $b=L$, we get

$$
\begin{gathered}
\xi_{T_{c}}\left(u, L^{-1}\right) \sim L^{\mathrm{\rho}} \Xi\left(A L^{2-d / 2} u^{1 / 2}\right), \\
g_{T_{c}}\left(u, \mathbf{x}, L^{-1}\right) \sim L^{-d / 2} G\left(A L^{2-d / 2} u^{1 / 2}\right) .
\end{gathered}
$$

Above the upper critical dimension, $Q=d / d_{c}$ and the notion of thermodynamic length is abandoned in the QFSS picture [18-21]. Below $d_{c}, Q=1$ and ordinary FSS is recovered.

This picture is corroborated by analytical and numerical calculations for systems with periodic boundary conditions (PBCs), both in the short-range Ising model (SRIM) above $d_{c}=4$ and in the long-range Ising model (LRIM) above $d_{c}=2 \sigma$ [18-21]. In the latter case, the general discussion has to be modified. The sum over interactions in Eq. (6) is extended to all pairs with decaying couplings $J_{\mathbf{x}-\mathbf{x}^{\prime}} \sim J /\left|\mathbf{x}-\mathbf{x}^{\prime}\right|^{d+\sigma}$. The MFT critical exponents of Eqs. (4) and (5) remain valid, except that $\nu=1 / \sigma$ and $\eta=2-\sigma$, and the RG eigenvalues at the Gaussian FP take the form $y_{t}=\sigma, y_{h}=(d+\sigma) / 2$, and $y_{u}=2 \sigma-d$ [22]. One recovers the SRIM values with $\sigma=2$. The standard scaling laws (12) are satisfied above $d_{c}$, while those of (11) are not, indicating again the dangerous irrelevancy of the quartic term in the action, which now contains an additional $|\mathbf{k}|^{\sigma}$ contribution. The modified (starred) RG dimensions are the same as before. The agreement between numerical results for PBCs and the scaling picture of Eqs. (17), (18), (21), and (22) is complete when simulations are performed at $T_{c}$, but also when they are performed at the pseudocritical point $T_{L}$ (defined by the size-dependent temperature where a quantity such as the susceptibility exhibits a maximum).

When free boundary conditions (FBCs) are imposed, an intriguing feature appears. Simulation results are consistent with the above picture at the pseudocritical point $T_{L}$ of the FBC system [19], but not at $T_{c}$, where, instead, standard FSS with the Landau MFT-type exponents of Eq. (5) has been obtained for the susceptibility [23-27].

As recently shown by Wittman and Young [24] (see also Refs. [10,11,23]), the Fourier modes play a key role. The nonzero modes, which are not affected by DIVs, contribute to the FSS of the susceptibility with the Landau ratio $\gamma / \nu=2$ for PBCs at both $T_{c}$ and $T_{L}$, and they argued for the same Landau behavior of analogous modes for FBCs at $T_{L}$. We show below that standard FSS with Landau exponents is not correct for all thermodynamic functions; it is in conflict with the RG.

We follow Ref. [23] and perform a sine expansion of the scalar field in Eq. (7) satisfying $\phi(\mathbf{x})=0$ at the free surfaces: $\phi(\mathbf{x})=\sum_{\mathbf{k}} \phi_{\mathbf{k}} \prod_{\alpha=1}^{d} \sqrt{2 / L} \sin k_{\alpha} x_{\alpha}$, where $k_{\alpha}=n_{\alpha} \pi / L, n_{\alpha}=1,2, \ldots, L$. In $\mathbf{k}$ space, the action takes a form slightly different than that for PBCs, and one must distinguish modes for which all $n_{\alpha}$ values are odd integers. These are analogous to the zero mode in the PBC case, and we denote their set by $\mathcal{Q}$. We denote the remaining modes by $\mathcal{G}$. The action now reads [23]

$$
\begin{aligned}
S[\phi]= & \frac{1}{2} \sum_{\mathbf{k}}\left(r_{0}+c|\mathbf{k}|^{2}\right) \phi_{\mathbf{k}}^{2}-\left(\frac{8}{L}\right)^{d / 2} h \sum_{\mathbf{k} \in \mathcal{Q}} \phi_{\mathbf{k}} \prod_{j=1}^{d} \frac{1}{k_{j}} \\
& +\frac{u}{L^{d}} \sum_{\mathbf{k}_{1}, \mathbf{k}_{2}, \mathbf{k}_{3}, \mathbf{k}_{4}} \Delta_{\mathbf{k}_{1}, \mathbf{k}_{2}, \mathbf{k}_{3}, \mathbf{k}_{4}} \phi_{\mathbf{k}_{1}} \phi_{\mathbf{k}_{2}} \phi_{\mathbf{k}_{3}} \phi_{\mathbf{k}_{4}},
\end{aligned}
$$

where the $\Delta_{i}$ 's are momentum-conserving factors. The difference between the quadratic terms of Eqs. (13) and (23) is the source for the difference in scaling between the pseudocritical shifts in the PBC and FBC cases [23]. The quartic term in Eq. (23) is dangerous only for the modes $\mathbf{k} \in \mathcal{Q}$, which couple to $h$. We henceforth refer to modes for which $u$ is dangerous (in particular, the zero mode at $T_{c}$ and $T_{L}$ for PBCs and modes with all odd $n_{\alpha}$ 's at $T_{L}$ for FBCs) as $Q$ modes and the remaining ones as Gaussian modes or $G$ modes.

We introduce the notation $m_{\mathbf{k}}$ to represent the contribution of a single mode $\mathbf{k}$ to the average magnetization. Thus, $m_{\mathbf{k}}=\left\langle\phi_{\mathbf{k}}\right\rangle=\left\langle\int d^{d} x \phi(\mathbf{x}) \psi_{\mathbf{k}}(\mathbf{x})\right\rangle$, where $\psi_{\mathbf{k}}(\mathbf{x})$ is the standing wave or the sine mode, depending upon the BCs. The brackets indicate the thermal average with the Boltzmann weight corresponding to the action (13) or (23). The equilibrium magnetization is then $m=\left\langle\int d^{d} x \sum_{\mathbf{k}} \phi_{\mathbf{k}} \psi_{\mathbf{k}}(\mathbf{x})\right\rangle$. The $Q$ modes acquire nonvanishing expectation values and have projections onto the equilibrium magnetization $m[23,24]$. The $G$ modes do not have such projections and were expected, in the customary picture, to exhibit standard FSS given by Eq. (3) with Landau exponents (5). This was supported by numerical evidence for the susceptibility Fourier modes $\chi_{\mathbf{k} \in \mathcal{Q}}$ in Ref. [24].

This standard picture for $G$ modes is incorrect, as we shall now see. Either we study physical quantities which are related to the $Q$ modes, in which case the DIV has to be properly taken into account, or we analyze properties associated with $G$ modes for which $u$ is not dangerous. In the latter case the exponents are those predicted by the RG at the Gaussian FP; these are (11) and (12), and not Landau exponents (4) and (5). In the first case, on the other hand, the exponents are indeed MFT exponents (4) and (5), but the correlation length has the FSS behavior involving $Q$, and Eq. (3) has to be modified to QFSS, which, e.g., for magnetization and susceptibility in the appropriate scaling regime, reads as

$$
\begin{aligned}
& m\left(t, L^{-1}\right) \sim|t|^{\beta}\left[L^{\mathrm{Q}} / \xi(t)\right]^{-\beta / \nu} \sim L^{-\mathrm{Q} \beta / \nu} \sim L^{-d / 4}, \\
& \chi\left(t, L^{-1}\right) \sim|t|^{-\gamma}\left[L^{\mathrm{Q}} / \xi(t)\right]^{\gamma / \nu} \sim L^{\mathrm{Q} \gamma / \nu} \sim L^{d / 2} .
\end{aligned}
$$

The $Q$ modes $m_{\mathbf{k} \in \mathcal{Q}}$ and $\chi_{\mathbf{k} \in \mathcal{Q}}$ themselves also obey QFSS. For $G$ modes this reduces to standard FSS: 
TABLE I. The partitioning of Fourier modes into dangerous $(\mathcal{Q})$ and nondangerous $(\mathcal{G})$ sectors of the model and FSS forms supported by Figs. 1 and 2. The Landau FSS form $m \sim L^{-\beta / \nu}=L^{-\sigma / 2}$ is not supported in the figures.

\begin{tabular}{|c|c|c|c|c|}
\hline & \multicolumn{2}{|c|}{ PBC } & \multicolumn{2}{|c|}{$\mathrm{FBC}$} \\
\hline & \multicolumn{2}{|c|}{$k=(2 \pi / L)\left(n_{1}, \ldots, n_{d}\right)$} & \multicolumn{2}{|c|}{$k=[\pi /(L+1)]\left(n_{1}, \ldots, n_{d}\right)$} \\
\hline & $\begin{array}{l}\mathcal{Q}(\mathrm{DIV}): \\
\text { all } n_{\alpha}=0\end{array}$ & $\begin{array}{l}\mathcal{G}(\text { non-DIV): } \\
\text { any } n_{\alpha} \neq 0\end{array}$ & $\begin{array}{l}\mathcal{Q} \text { (DIV): } \\
\text { all } n_{\alpha} \text { odd }\end{array}$ & $\begin{array}{l}\mathcal{G} \text { (non-DIV): } \\
\text { any } n_{\alpha} \text { even }\end{array}$ \\
\hline FSS & $m \sim L^{-d / 4}$ & $m \sim L^{-(d-\sigma) / 2}$ & $m \sim L^{-d / 4}$ & $m \sim L^{-(d-\sigma) / 2}$ \\
\hline at $T_{L}$ & $\chi \sim L^{d / 2}$ & $\chi \sim L^{\sigma}$ & $\chi \sim L^{d / 2}$ & $\chi \sim L^{\sigma}$ \\
\hline FSS & $m \sim L^{-d / 4}$ & $m \sim L^{(d-\sigma) / 2}$ & $m \sim L^{-(d-\sigma) / 2}$ & $m \sim L^{-(d-\sigma) / 2}$ \\
\hline at $T_{c}$ & $\chi \sim L^{d / 2}$ & $\chi \sim L^{\sigma}$ & $\chi \sim L^{\sigma}$ & $\chi \sim L^{\sigma}$ \\
\hline
\end{tabular}

$m_{\mathbf{k} \in \mathcal{G}}\left(t, L^{-1}\right) \sim|t|^{\left(d-y_{h}\right) / y_{t}}[L / \xi(t)]^{-\left(d-y_{h}\right)} \sim L^{-(d-\sigma) / 2}$,

$$
\chi_{\mathbf{k} \in \mathcal{G}}\left(t, L^{-1}\right) \sim|t|^{-\left(2 y_{h}-d\right) / y_{t}}[L / \xi(t)]^{2 y_{h}-d} \sim L^{\sigma} .
$$

Equation (27) agrees numerically with the result of Wittmann and Young [24] because the combination of exponents $2 y_{h}-d$ equals $\sigma$ and coincides with $\gamma / \nu$ (for the SRIM, $\sigma=2$ ). However, there is no way to reconcile Eq. (26) with the power $-\beta / \nu=-\sigma / 2(=-1$ for the SRIM) for an arbitrary value of $d$ above $d_{c}=2 \sigma$.

In order to determine which of the forms (i) Eq. (24) from QFSS, (ii) Eq. (26) from the Gaussian FP, or even (iii) $m \sim L^{-\beta / \nu}$ from the Landau exponents in Eq. (3) correctly describes the scaling of the magnetization at $T_{c}$, we performed Monte Carlo simulations for the SRIM and the LRIM above $d_{c}$, along the lines described in Ref. [21]. Since we are simulating with finite-size lattices, we examine the magnitudes of the Fourier-mode contributions through $m_{\mathbf{k}}\left(L^{-1}\right)=\left\langle\left|s_{\mathbf{k}}\right|\right\rangle=\left\langle\left|\sum_{\mathbf{x}} s_{\mathbf{x}} \psi_{\mathbf{k}}(\mathbf{x})\right|\right\rangle$, where $s_{\mathbf{X}}$ is an Ising spin at site $\mathbf{x}=a \mathbf{n}$. The total magnetization is $m\left(L^{-1}\right)=\left\langle\left|\sum_{\mathbf{x}} s_{\mathbf{x}}\right|\right\rangle$.

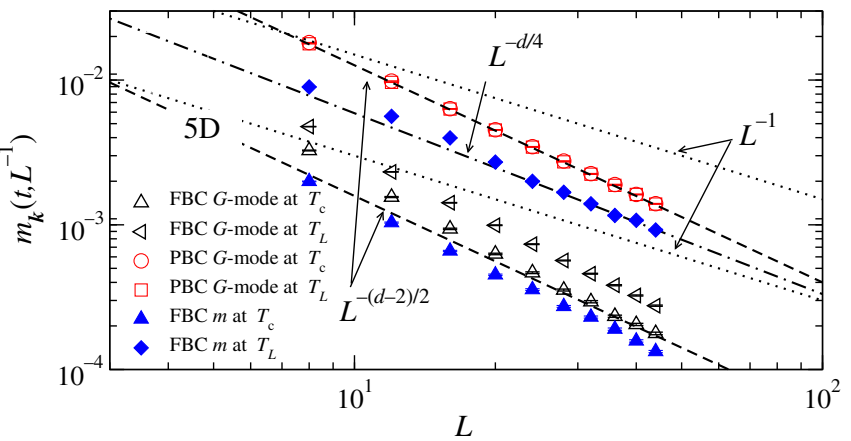

FIG. 1. FSS for the 5D SRIM. The magnetization for FBCs belongs to the DIV sector and scales as $m\left(T_{L}\right) \sim L^{-d / 4}$ (filled diamonds). At $T_{c}$ the magnetization scales as $L^{-(d-2) / 2}$, as predicted by Gaussian-FP FSS (filled triangles). The remaining data are for the lowest critical and pseudocritical $G$ modes $m_{\mathbf{k}}\left(L^{-1}\right)$, each of which belongs to the non-DIV sector. Here, we see they also follow Gaussian-FP FSS and not the Landau FSS prediction $L^{-1}$ (which is indicated by the dotted lines).
The various sectors examined and outcomes supported are summarized in Table I. For the DIV sectors, Eq. (24) gives $m\left(L^{-1}\right) \sim L^{-d / 4}$. For PBCs at $T_{c}$, this is already well established, having also been verified numerically in Ref. [28]. We find the same behavior for $Q$ modes for PBCs and FBCs at $T_{L}$ for both the five-dimensional (5D) SRIM and the one-dimensional (1D) LRIM. For the nonDIV sectors, standard FSS with Landau exponents predicts $m\left(L^{-1}\right) \sim L^{-\sigma / 2}$, while we predict $m\left(L^{-1}\right) \sim L^{-(d-\sigma) / 2}$ after Eq. (26). For the 5D SRIM, then, a plot on a double logarithmic scale should have slope -1 according to "Landau FSS," or $-3 / 2$ according to Eq. (26). Our results for $G$ modes in Fig. 1 are clearly compatible with the latter and rule out the former. For the LRIM above $d_{c}$, the outcome is even more stark; Landau FSS predicts a slope of $-\sigma / 2=-0.05$, independent of $d$, and Eq. (26) predicts $-(d-\sigma) / 2=-0.45$, for $d=1$, the latter being clearly favored by the data in Fig. 2.

The results also confirm that the above-mentioned feature associated with FBCs extends to other $Q$-mode quantities; while they follow Eqs. (24) and (25) at $T_{L}$, they do not at $T_{c}$ [23-27]. However, the results furthermore show that they also obey scaling given by Eqs. (26)

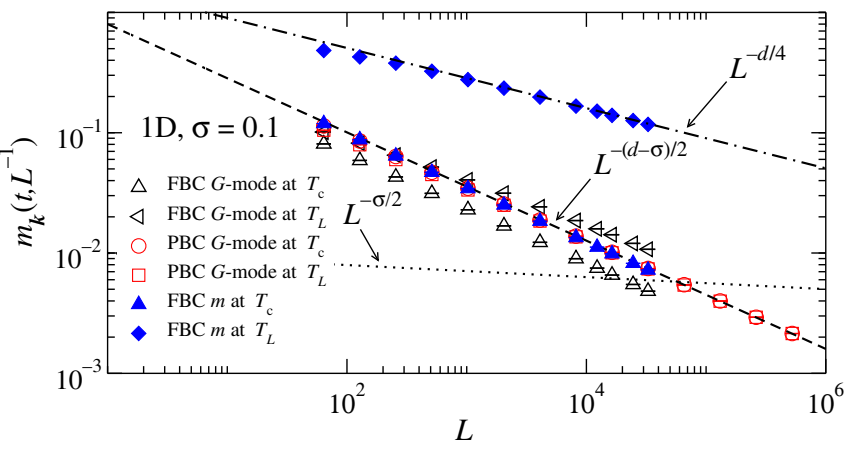

FIG. 2. FSS for the 1D LRIM with $\sigma=0.1$. As in Fig. 1, the magnetization for FBCs exhibits QFSS at $T_{L}$, scaling as $m\left(T_{L}\right) \sim$ $L^{-d / 4}$ (filled diamonds), and Gaussian-FP FSS at $T_{c}$, scaling as $L^{-(d-\sigma) / 2}$ (filled triangles). The lowest critical and pseudocritical $G$ modes all also follow Gaussian-FP FSS and not the Landau FSS prediction $L^{-\sigma / 2}$ (which is indicated by the dotted line). 
and (27) like $G$ quantities, rather than standard FSS with Landau exponents.

In summary, above the upper critical dimension, the numerical evidence clearly rules out Landau FSS for $G$ modes and supports a direct physical manifestation of Gaussian-FP exponents there, unaffected by DIVs. $Q$ modes for FBCs also exhibit $G$-like scaling at $T_{c}$ rather than Landau FSS.

In the literature, it has been stated that "due to the lack of a better way of treating the zero-momentum modes," it is usual to "neglect them completely" [29]. Standard phenomenological FSS associated with the excited modes was then expected to deliver Landau FSS. The results established here indicate that this approach is not correct. Also in the literature, the critical exponents (11) and (12) are presented as nonphysical; they were hitherto merely a step on the way to reconciling RG theory with Landau mean-field exponents above $d_{c}$. Here, we have shown that they are, in fact, physically manifest in the magnitudes of the $G$ modes. This is not a feature of finite size only. In the thermodynamic limit, $G$ modes should, for example, acquire the temperature behavior $m_{\mathbf{k} \in \mathcal{G}}(t) \sim|t|^{d / 2 \sigma-1 / 2}$. Moreover, mean-field theory fails to fully describe either $G$ or $Q$ modes above the upper critical dimension. Instead, this careful approach to RG, taking QFSS into account, delivers a consistent description for all Fourier modes.

This work was supported by the EU FP7 Projects No. 269139, "Dynamics and Cooperative Phenomena in Complex Physical and Biological Media," No. 295302, "Statistical Physics in Diverse Realizations," and No. 612707, "Dynamics of and in Complex Systems," and by the Doctoral College for the Statistical Physics of Complex Systems, Leipzig-Lorraine-Lviv-Coventry, Franco-German University.

[1] K. G. Wilson, Renormalization group and critical phenomena. I. Renormalization group and the Kadanoff scaling picture, Phys. Rev. B 4, 3174 (1971); Renormalization group and critical phenomena. II. Phase-space cell analysis of critical behavior, scaling picture, Phys. Rev. B, 4, 3184 (1971).

[2] M. E. Fisher, in Critical Phenomena, Proceedings of the 51st Enrico Fermi Summer School, Varenna, Italy, edited by M. S. Green (Academic Press, New York, 1971), p. 1.

[3] K. Wilson and J. B. Kogut, The renormalization group and the $\epsilon$ expansion, Phys. Rep. 12, 75 (1974).

[4] S. K. Ma, Modern Theory of Critical Phenomena (Westview Press, New York, 1976).

[5] E. Brézin, An investigation of finite size scaling, J. Phys. (Paris) 43, 15 (1982).

[6] R. B. Griffiths, Dependence of Critical Indices on a Parameter, Phys. Rev. Lett. 24, 1479 (1970).

[7] M. E. Fisher, in Critical Phenomena, Lecture Notes in Physics Vol. 186, edited by F. J. W. Hahne (Springer, Berlin, 1983), p. 1.
[8] J. L. Cardy, Scaling and Renormalization in Statistical Physics (Cambridge University Press, Cambridge, 1996), p. 93.

[9] E. Brézin and J. Zinn-Justin, Finite size effects in phase transitions, Nucl. Phys. B257, 867 (1985).

[10] E. Luijten and H. W. J. Blöte, Finite-Size Scaling and Universality above the Upper Critical Dimension, Phys. Rev. Lett. 76, 1557 (1996); 76, 3662(E) (1996).

[11] K. Binder and E. Luijten, Monte Carlo tests of renormalization group predictions for critical phenomena in Ising models, Phys. Rep. 344, 179 (2001).

[12] K. Binder, M. Nauenberg, V. Privman, and A. P. Young, Finite size tests of hyperscaling, Phys. Rev. B 31, 1498 (1985).

[13] K. Binder, Critical properties and finite-size effects of the five-dimensional Ising model, Z. Phys. B 61, 13 (1985).

[14] V. Privman, in Finite Size Scaling and Numerical Simulation of Statistical Systems, edited by V. Privman (World Scientific, Singapore, 1990), p. 4.

[15] P. M. Chaikin and T. C. Lubensky, Principles of Condensed Matter Physics (Cambridge University Press, Cambridge, England, 2000).

[16] P. Kopietz, L. Bartosch, and F. Schütz, Introduction to the Functional Renormalization Group, Lecture Notes in Physics Vol. 798 (Springer-Verlag, Berlin, 2010).

[17] H. Nishimori, Elements of Phase Transitions and Critical Phenomena (Oxford University Press, Oxford, 2011).

[18] R. Kenna and B. Berche, Fisher's scaling relation above the upper critical dimension, Europhys. Lett. 105, 26005 (2014).

[19] B. Berche, R. Kenna, and J. C. Walter, Hyperscaling above the upper critical dimension, Nucl. Phys. B865, 115 (2012).

[20] R. Kenna and B. Berche, A new critical exponent $Q$ and its logarithmic counterpart $\hat{Q}$, Condens. Matter Phys. 16, 23601 (2013).

[21] E. J. Flores-Sola, B. Berche, R. Kenna, and M. Weigel, Finite-size scaling above the upper critical dimension in Ising models with long-range interactions, Eur. Phys. J. B 88, 28 (2015).

[22] M. E. Fisher, S. K. Ma, and B. G. Nickel, Critical Exponents for Long-Range Interactions, Phys. Rev. Lett. 29, 917 (1972).

[23] J. Rudnick, G. Gaspari, and V. Privman, Effect of boundary conditions on critical behavior of a finite high-dimensional Ising model, Phys. Rev. B 32, 7594 (1985).

[24] M. Wittmann and A. P. Young, Finite-size scaling above the upper critical dimension, Phys. Rev. E 90, 062137 (2014).

[25] P. G. Watson in Phase Transitions and Critical Phenomena, Vol. 2, edited by C. Domb and M. S. Green (Academic, London, 1973), p. 101.

[26] P. H. Lundow and K. Markström, Non-vanishing boundary effects and quasifirst-order phase transitions in high dimensional Ising models, Nucl. Phys. B845, 120 (2011).

[27] P. H. Lundow and K. Markström, Finite size scaling of the 5D Ising model with free boundary conditions, Nucl. Phys. B889, 249 (2014).

[28] G. Parisi and J. J. Ruiz-Lorenzo, Scaling above the upper critical dimension in Ising models, Phys. Rev. B 54, R3698 (1996); N. Aktekin and Ş. Erkoç, The test of the finite-size 
scaling relations for the six-dimensional Ising model on the Creutz cellular automato, Physica (Amsterdam) 284A, 206 (2000); The test of the finite-size scaling relations for the seven-dimensional Ising model on the Creutz cellular automaton, Physica (Amsterdam), 290A, 123 (2001); Z. Merdan and R. Erdem, The finite-size scaling study of the specific heat and the Binder parameter for the six-dimensional Ising model, Phys. Lett. A 330, 403 (2004); Z. Merdan and M. Bayrih, The effect of the increase of linear dimensions on exponents obtained by finite-size scaling relations for the six dimensional Ising model on the Creutz cellular automaton, Applied Mathematics and Computation 167, 212 (2005); Z. Merdan, A. Duran, D. Atille, G. Mülazimoğlu, and A. Günen, The test of the finite-size scaling relations of the Ising models in seven and eight dimensions on the Creutz cellular automaton, Physica (Amsterdam) 366A, 265 (2006).

[29] U. Heller and F. Karsch, One-loop perturbative calculation of Wilson loops on finite lattices, Nucl. Phys. B251, 254 (1985). 